\title{
Existence and uniqueness of periodic solutions of second-order nonlinear differential equations
}

Renwei Jia ${ }^{1}$ and Jianying Shao ${ }^{2^{*}}$

"Correspondence:
shaojianying2008@yahoo.cn
${ }^{2}$ College of Mathematics, Physics
and Information Engineering,
Jiaxing University, Jiaxing, Zhejiang
314001, P.R. China
Full list of author information is
available at the end of the article

*Correspondence:

shaojianying2008@yahoo.cn and Information Engineering, 314001, P.R. China

available at the end of the article

\begin{abstract}
This paper is concerned with the following second-order nonlinear differential equation:

$$
x^{(2 n)}(t)+\sum_{k=1}^{n+1} f_{k}\left(x^{(k-1)}(t)\right) x^{(k)}(t)-g(t, x(t))=e(t) .
$$

By applying Mawhin's continuation theorem of coincidence degree theory, we establish sufficient conditions for the existence and uniqueness of periodic solutions for the above equation. Some recent results are known as the special cases of ours.
\end{abstract}

Keywords: second-order nonlinear differential equations; periodic solutions; Mawhin's continuation theorem

\section{Introduction}

In applied science, some practical problems such as nonlinear oscillations [1, 2], fluid mechanical and nonlinear elastic mechanical phenomena [3-9] are associated with the periodic solutions of nonlinear high-order differential equations. Recently, Bereanu [10], Zhao et al. [11] and Fan et al. [12] investigated the existence of $T$-periodic solutions for the following fourth-order nonlinear differential equation:

$$
x^{\prime \prime \prime \prime}(t)+a x^{\prime \prime \prime}(t)-p x^{\prime \prime}(t)+q x^{\prime}(t)-g(t, x(t))=e(t) .
$$

Xu et al. [13] dealt with the existence of $T$-periodic solutions for the second-order nonlinear differential equation as follows:

$$
x^{(2 n)}(t)+h\left(x^{(n)}(t)\right) x^{(n+1)}(t)-P\left(x^{\prime}(t)\right) x^{\prime \prime}(t)+f(x(t)) x^{\prime}(t)-g(t, x(t))=e(t),
$$

where $n \geq 2$ is an even integer, $h, P, f, e: R \rightarrow R$ and $g: R^{2} \rightarrow R$ represent continuous functions, $e$ and $g$ respectively denote the $T$-periodic and $T$-periodic in the first argument and $T>0$. However, to the best of our knowledge, most authors mentioned above have only considered the existence of periodic solutions of Eqs. (1.1) and (1.2). There are still few studies on the uniqueness of periodic solutions for these equations. Thus, in this case, it 
is worth investigating both the existence and the uniqueness of periodic solutions for a high-order nonlinear differential equation.

In this paper, we study the existence and uniqueness of $T$-periodic solutions for the second-order nonlinear differential equation

$$
x^{(2 n)}(t)+\sum_{k=1}^{n+1} f_{k}\left(x^{(k-1)}(t)\right) x^{(k)}(t)-g(t, x(t))=e(t)
$$

where $n \geq 2$ is an even integer, $f_{k}, e: R \rightarrow R$ and $g: R^{2} \rightarrow R$ represent continuous functions and $f_{k}(k=1,2, \ldots, n+1)$ are bounded, $e$ and $g$ respectively denote the $T$-periodic and $T$-periodic in the first argument and $T>0$.

Obviously, Eq. (1.1) is a special case of Eq. (1.3) with $n=2, f_{3}(x) \equiv a, f_{2}(x) \equiv-p$ and $f_{1}(x) \equiv q$. Moreover, Eq. (1.2) is another special case of Eq. (1.3) with $f_{3}(x)=f_{4}(x)=\cdots=$ $f_{n+1}(x) \equiv 0$

For ease of exposition, we will adopt the following notations throughout this paper:

$$
\begin{aligned}
& F_{k}(x)=\int_{0}^{x} f_{k}(s) d s, \quad k=1,2, \ldots, n+1, \text { for all } x \in R, \\
& \|x\|_{2}=\left(\int_{0}^{T}|x(t)|^{2} d t\right)^{1 / 2}, \quad\|x\|_{\infty}=\max _{t \in[0, T]}|x(t)|, \\
& L_{k}=\sup _{s \in R}\left|f_{k}(s)\right|, \quad k=1,2, \ldots, n+1 .
\end{aligned}
$$

Let

$$
C_{T}^{m}=\left\{x \mid x \in C^{m}(R, R), x(t+T)=x(t) \text { for all } t \in R, m \in N\right\}
$$

be Banach spaces with the norms

$$
\|x\|_{(m)}=\sum_{k=0}^{m}\left\|x^{(k)}\right\|_{\infty} \quad\left(x \in C_{T}^{m}\right)
$$

To obtain our results, we also make the following assumptions:

(S1) There exists $d>0$ such that, for any continuous $T$-periodic function $x$, we have

$$
[g(t, x(t))+e(t)] x(t)>0 \quad \text { for all } t \in R,|x(t)| \geq d
$$

or

$$
[g(t, x(t))+e(t)] x(t)<0 \quad \text { for all } t \in R,|x(t)| \geq d
$$

(S2) For $t, x_{1}, x_{2} \in R, x_{1} \neq x_{2}$,

$$
\left[g\left(t, x_{1}\right)-g\left(t, x_{2}\right)\right]\left(x_{1}-x_{2}\right)<0, \quad 1>L_{1} \frac{T}{\pi}\left(\frac{T}{2 \pi}\right)^{2(n-1)}+\sum_{k=2}^{n+1} L_{k}\left(\frac{T}{2 \pi}\right)^{2 n-k} .
$$


(S3) For $t, x_{1}, x_{2} \in R, x_{1} \neq x_{2}$, there exists a nonnegative constant $B$ such that

$$
B\left(x_{1}-x_{2}\right)^{2} \geq\left[g\left(t, x_{1}\right)-g\left(t, x_{2}\right)\right]\left(x_{1}-x_{2}\right)>0,
$$

and

$$
1>L_{1} \frac{T}{\pi}\left(\frac{T}{2 \pi}\right)^{2(n-1)}+\sum_{k=2}^{n+1} L_{k}\left(\frac{T}{2 \pi}\right)^{2 n-k}+B\left(\frac{T}{\pi}\right)^{2}\left(\frac{T}{2 \pi}\right)^{2(n-1)} .
$$

\section{Preliminaries}

The following lemmas will be useful to prove our main results in Section 3.

Let $\tilde{f}: R^{2 n+1} \rightarrow R$ be a continuous function, $T$-periodic with respect to the first variable, and consider the second-order differential equation

$$
x^{(2 n)}=\tilde{f}\left(t, x, x^{\prime}, x^{\prime \prime}, \ldots, x^{(2 n-1)}\right)
$$

Lemma 2.1 (See [14]) Assume that the following conditions hold.

(i) There exists $\rho>0$ such that, for each $\lambda \in(0,1]$, one has that any possible $T$-periodic solution $x$ of the problem

$$
x^{(2 n)}=\lambda \tilde{f}\left(t, x, x^{\prime}, x^{\prime \prime}, \ldots, x^{(2 n-1)}\right)
$$

satisfies the a priori estimation $\|x\|_{(2 n-1)}<\rho$.

(ii) The continuous function $F: R \rightarrow R$ defined by

$$
F(x)=\int_{0}^{T} \tilde{f}(t, x, 0,0, \ldots, 0) d t, \quad x \in R
$$

satisfies $F(-\rho) F(\rho)<0$.

Then (2.1) has at least one T-periodic solution $x$ such that $\|x\|_{(2 n-1)}<\rho$.

From Lemma 2.2 in [15] and the proof of inequality (10) in [12, p.124], we obtain the following.

Lemma 2.2 Let $x(t) \in C_{T}^{1}$. Suppose that there exist two constants $D>0, t_{0} \in[0, T]$ such that $\left|x\left(t_{0}\right)\right| \leq D$, then

$$
\|x\|_{2} \leq \frac{T}{\pi}\left\|x^{\prime}\right\|_{2}+\sqrt{T} D, \quad\|x\|_{\infty} \leq D+\frac{1}{2} \sqrt{T}\left\|x^{\prime}\right\|_{2} .
$$

Lemma 2.3 (See $[16,17])$ If $x \in C_{T}^{2}$, then

$$
\left\|x^{\prime}\right\|_{2}^{2} \leq\left(\frac{T}{2 \pi}\right)^{2}\left\|x^{\prime \prime}\right\|_{2}^{2}
$$

Lemma 2.4 (See [13]) For any $x \in C_{T}^{n}$, one has that

$$
\left\|x^{(k)}\right\|_{\infty} \leq T^{(n-1)-k}\left(\frac{1}{2}\right)^{(n-1)-(k-1)} \int_{0}^{T}\left|x^{(n)}(t)\right| d t, \quad k=1,2, \ldots, n-1 .
$$


Now, let

$$
\begin{aligned}
& \tilde{f}\left(t, x, x^{\prime}, x^{\prime \prime}, \ldots, x^{(2 n-1)}\right) \\
& \quad=g(t, x(t))+e(t)-\sum_{k=1}^{n+1} f_{k}\left(x^{(k-1)}(t)\right) x^{(k)}(t) .
\end{aligned}
$$

For $\lambda \in(0,1]$, we consider the second-order differential equation

$$
\begin{aligned}
x^{(2 n)}(t) & =\lambda \tilde{f}\left(t, x, x^{\prime}, x^{\prime \prime}, \ldots, x^{(2 n-1)}\right) \\
& =\lambda[g(t, x(t))+e(t)]-\lambda \sum_{k=1}^{n+1} f_{k}\left(x^{(k-1)}(t)\right) x^{(k)}(t) .
\end{aligned}
$$

Lemma 2.5 Suppose that (S1), (S2) (or (S3)) hold, then there exists a fixed constant $C^{*}>0$ independent of $\lambda$ and $x$ such that any possible T-periodic solution $x$ of $(2.5)$ satisfies

$$
\left\|x^{(k)}\right\|_{\infty} \leq C^{*}, \quad k=0,1,2, \ldots, n-1
$$

Proof Let $\lambda \in(0,1]$ and let $x$ be a possible $T$-periodic solution of (2.5). Integrating (2.5) from 0 to $T$ yields

$$
\int_{0}^{T}[g(t, x(t))+e(t)] d t=0
$$

which together with (S1) implies that

$$
\exists \xi \in[0, T]:|x(\xi)|<d
$$

In view of (2.2) and (2.3), we get

$$
\|x\|_{\infty} \leq d+\frac{1}{2} \sqrt{T}\left\|x^{\prime}\right\|_{2} \leq d+\frac{T \sqrt{T}}{4 \pi}\left\|x^{\prime \prime}\right\|_{2}
$$

Thus

$$
\begin{aligned}
& \left|\int_{0}^{T} e(t) x(t) d t\right| \leq\|e\|_{\infty}\|x\|_{\infty} T \leq T\|e\|_{\infty} d+\frac{T^{2} \sqrt{T}}{4 \pi}\|e\|_{\infty}\left\|x^{\prime \prime}\right\|_{2}, \\
& \left|\int_{0}^{T} g(t, 0) x(t) d t\right| \leq T\|g(t, 0)\|_{\infty} d+\frac{T^{2} \sqrt{T}}{4 \pi}\|g(t, 0)\|_{\infty}\left\|x^{\prime \prime}\right\|_{2} .
\end{aligned}
$$

On the other hand, multiplying Eq. (2.5) by $x$ and integrating it from 0 to $T$, we obtain

$$
\begin{aligned}
\left\|x^{(n)}\right\|_{2}^{2} & =\int_{0}^{T}\left|x^{(n)}(t)\right|^{2} d t \\
& =\lambda \sum_{k=2}^{n+1} \int_{0}^{T} F_{k}\left(x^{(k-1)}(t)\right) x^{\prime}(t) d t+\lambda \int_{0}^{T} g(t, x(t)) x(t) d t+\lambda \int_{0}^{T} e(t) x(t) d t .
\end{aligned}
$$


Combining (2.2), (2.3), (2.8) and (2.9), we obtain

$$
\begin{aligned}
& \left\|x^{(n)}\right\|_{2}^{2}=\int_{0}^{T}\left|x^{(n)}(t)\right|^{2} d t \\
& =\lambda \sum_{k=2}^{n+1} \int_{0}^{T}\left[F_{k}\left(x^{(k-1)}(t)\right)-F_{k}(0)\right] x^{\prime}(t) d t \\
& +\lambda \int_{0}^{T}[g(t, x(t))-g(t, 0)][x(t)-0] d t \\
& +\lambda \int_{0}^{T} g(t, 0) x(t) d t+\lambda \int_{0}^{T} e(t) x(t) d t \\
& \leq \sum_{k=2}^{n+1} L_{k} \int_{0}^{T}\left|x^{(k-1)}(t)\right|\left|x^{\prime}(t)\right| d t+\left|\int_{0}^{T} g(t, 0) x(t) d t\right| \\
& +\left|\int_{0}^{T} e(t) x(t) d t\right|+\lambda \int_{0}^{T}[g(t, x(t))-g(t, 0)][x(t)-0] d t \\
& \leq \sum_{k=2}^{n+1} L_{k}\left(\int_{0}^{T}\left|x^{(k-1)}(t)\right|^{2} d t\right)^{\frac{1}{2}}\left(\int_{0}^{T}\left|x^{\prime}(t)\right|^{2} d t\right)^{\frac{1}{2}} \\
& +\left(\|e\|_{\infty}+\|g(t, 0)\|_{\infty}\right) \frac{T^{2} \sqrt{T}}{4 \pi}\left\|x^{\prime \prime}\right\|_{2} \\
& +\left(\|e\|_{\infty}+\|g(t, 0)\|_{\infty}\right) T d+\lambda \int_{0}^{T}[g(t, x(t))-g(t, 0)][x(t)-0] d t \\
& \leq \sum_{k=2}^{n+1} L_{k}\left(\frac{T}{2 \pi}\right)^{2 n-k}\left\|x^{(n)}\right\|_{2}^{2}+\left(\|e\|_{\infty}+\|g(t, 0)\|_{\infty}\right) \frac{T^{2} \sqrt{T}}{4 \pi}\left(\frac{T}{2 \pi}\right)^{n-2}\left\|x^{(n)}\right\|_{2} \\
& +\left(\|e\|_{\infty}+\|g(t, 0)\|_{\infty}\right) T d+\lambda \int_{0}^{T}[g(t, x(t))-g(t, 0)][x(t)-0] d t .
\end{aligned}
$$

Now suppose that (S2) (or (S3)) holds, we will consider two cases as follows.

Case (i). If (S2) holds, then

$$
\begin{aligned}
\left\|x^{(n)}\right\|_{2}^{2} \leq & \sum_{k=2}^{n+1} L_{k}\left(\frac{T}{2 \pi}\right)^{2 n-k}\left\|x^{(n)}\right\|_{2}^{2}+\left(\|e\|_{\infty}+\|g(t, 0)\|_{\infty}\right) \frac{T^{2} \sqrt{T}}{4 \pi}\left(\frac{T}{2 \pi}\right)^{n-2}\left\|x^{(n)}\right\|_{2} \\
& +\left(\|e\|_{\infty}+\|g(t, 0)\|_{\infty}\right) T d
\end{aligned}
$$

which implies that there exists a positive constant $C_{n}$ satisfying

$$
\int_{0}^{T}\left|x^{(n)}(t)\right|^{2} d t \leq C_{n} \quad \text { and } \quad \int_{0}^{T}\left|x^{(n)}(t)\right| d t \leq \sqrt{T C_{n}}
$$

Case (ii). If (S3) holds, (2.2) and (2.3) yield that

$$
\begin{aligned}
\left\|x^{(n)}\right\|_{2}^{2} & =\int_{0}^{T}\left|x^{(n)}(t)\right|^{2} d t \\
& \leq \sum_{k=2}^{n+1} L_{k}\left(\frac{T}{2 \pi}\right)^{2 n-k}\left\|x^{(n)}\right\|_{2}^{2}+\left(\|e\|_{\infty}+\|g(t, 0)\|_{\infty}\right) \frac{T^{2} \sqrt{T}}{4 \pi}\left(\frac{T}{2 \pi}\right)^{n-2}\left\|x^{(n)}\right\|_{2}
\end{aligned}
$$




$$
\begin{aligned}
& +\left(\|e\|_{\infty}+\|g(t, 0)\|_{\infty}\right) T d+B \int_{0}^{T}|x(t)|^{2} d t \\
\leq & \sum_{k=2}^{n+1} L_{k}\left(\frac{T}{2 \pi}\right)^{2 n-k}\left\|x^{(n)}\right\|_{2}^{2}+\left(\|e\|_{\infty}+\|g(t, 0)\|_{\infty}\right) \frac{T^{2} \sqrt{T}}{4 \pi}\left(\frac{T}{2 \pi}\right)^{n-2}\left\|x^{(n)}\right\|_{2} \\
& +\left(\|e\|_{\infty}+\|g(t, 0)\|_{\infty}\right) T d+B\left[\frac{T}{\pi}\left(\frac{T}{2 \pi}\right)^{n-1}\left\|x^{(n)}\right\|_{2}+\sqrt{T} d\right]^{2} \\
= & {\left[\sum_{k=2}^{n+1} L_{k}\left(\frac{T}{2 \pi}\right)^{2 n-k}+B\left(\frac{T}{\pi}\right)^{2}\left(\frac{T}{2 \pi}\right)^{2(n-1)}\right]\left\|x^{(n)}\right\|_{2}^{2}+\left[2 B d \frac{T \sqrt{T}}{\pi}\left(\frac{T}{2 \pi}\right)^{n-1}\right.} \\
& \left.+\left(\|e\|_{\infty}+\|g(t, 0)\|_{\infty}\right) \frac{T^{2} \sqrt{T}}{4 \pi}\left(\frac{T}{2 \pi}\right)^{n-2}\right]\left\|x^{(n)}\right\|_{2} \\
& +\left(\|e\|_{\infty}+\|g(t, 0)\|_{\infty}+B d\right) T d,
\end{aligned}
$$

which implies (2.10) holds.

Thus, from (2.2), (2.3), (2.4) and (2.10), we can choose a constant $C^{*}$ independent of $\lambda$ and $x$ such that

$$
\left\|x^{(k)}\right\|_{\infty} \leq C^{*}, \quad k=0,1,2, \ldots, n-1
$$

This completes the proof of Lemma 2.5.

Lemma 2.6 Suppose that (S2) (or (S3)) hold, then (1.3) has at most one T-periodic solution.

Proof Suppose that $x_{1}(t)$ and $x_{2}(t)$ are two $T$-periodic solutions of (1.3). Set $Z(t)=x_{1}(t)-$ $x_{2}(t)$. Then we obtain

$$
\begin{aligned}
& Z^{(2 n)}(t)+\sum_{k=1}^{n+1}\left[f_{k}\left(x_{1}^{(k-1)}(t)\right) x_{1}^{(k)}(t)-f_{k}\left(x_{2}^{(k-1)}(t)\right) x_{2}^{(k)}(t)\right] \\
& -\left[g\left(t, x_{1}(t)\right)-g\left(t, x_{2}(t)\right)\right]=0 .
\end{aligned}
$$

Integrating (2.11) from 0 to $T$ yields

$$
\int_{0}^{T}\left[g\left(t, x_{1}(t)\right)-g\left(t, x_{2}(t)\right)\right] d t=0 .
$$

Therefore, in view of the integral mean value theorem, it follows that there exists a constant $\gamma \in[0, T]$ such that

$$
g\left(\gamma, x_{1}(\gamma)\right)-g\left(\gamma, x_{2}(\gamma)\right)=0
$$

From (S2) (or (S3)), we get

$$
Z(\gamma)=x_{1}(\gamma)-x_{2}(\gamma)=0,
$$

which together with (2.2) implies

$$
\|Z\|_{2} \leq \frac{T}{\pi}\left\|Z^{\prime}\right\|_{2} .
$$


Multiplying (2.11) by $Z(t)$ and integrating it from 0 to $T$, we obtain

$$
\begin{aligned}
\left\|Z^{(n)}\right\|_{2}^{2}= & \int_{0}^{T}\left|Z^{(n)}(t)\right|^{2} d t \\
= & \sum_{k=1}^{n+1} \int_{0}^{T}\left[F_{k}\left(x_{1}^{(k-1)}(t)\right)-F_{k}\left(x_{2}^{(k-1)}(t)\right)\right] Z^{\prime}(t) d t \\
& +\int_{0}^{T}\left[g\left(t, x_{1}(t)\right)-g\left(t, x_{2}(t)\right)\right]\left[x_{1}(t)-x_{2}(t)\right] d t .
\end{aligned}
$$

Now suppose that (S2) (or (S3)) holds, we will consider two cases as follows.

Case (i). If (S2) holds, (2.3) and (2.13) yield that

$$
\begin{aligned}
\left\|Z^{(n)}\right\|_{2}^{2} & =\int_{0}^{T}\left|Z^{(n)}(t)\right|^{2} d t \\
& \leq \sum_{k=1}^{n+1} L_{k} \int_{0}^{T}\left|Z^{(k-1)}(t)\right|\left|Z^{\prime}(t)\right| d t \\
& \leq \sum_{k=1}^{n+1} L_{k}\left(\int_{0}^{T}\left|Z^{(k-1)}(t)\right|^{2} d t\right)^{\frac{1}{2}}\left(\int_{0}^{T}\left|Z^{\prime}(t)\right|^{2} d t\right)^{\frac{1}{2}} \\
& \leq\left[L_{1} \frac{T}{\pi}\left(\frac{T}{2 \pi}\right)^{2(n-1)}+\sum_{k=2}^{n+1} L_{k}\left(\frac{T}{2 \pi}\right)^{2 n-k}\right]\left\|Z^{(n)}\right\|_{2}^{2},
\end{aligned}
$$

which together with (2.3) and (2.12) implies that

$$
Z^{(n)}(t) \equiv Z^{(n-1)}(t) \equiv \cdots \equiv Z^{\prime}(t) \equiv Z(t) \equiv 0 \quad \text { for all } t \in R
$$

Hence, Eq. (1.3) has at most one $T$-periodic solution.

Case (ii). If (S3) holds, (2.3) and (2.13) yield that

$$
\begin{aligned}
\left\|Z^{(n)}\right\|_{2}^{2}= & \int_{0}^{T}\left|Z^{(n)}(t)\right|^{2} d t \\
\leq & \sum_{k=1}^{n+1} L_{k} \int_{0}^{T}\left|Z^{(k-1)}(t)\right|\left|Z^{\prime}(t)\right| d t+B \int_{0}^{T}|Z(t)|^{2} d t \\
\leq & B\left(\frac{T}{\pi}\right)^{2}\left(\frac{T}{2 \pi}\right)^{2(n-1)} \int_{0}^{T}\left|Z^{(n)}(t)\right|^{2} d t \\
& +\sum_{k=1}^{n+1} L_{k}\left(\int_{0}^{T}\left|Z^{(k-1)}(t)\right|^{2} d t\right)^{\frac{1}{2}}\left(\int_{0}^{T}\left|Z^{\prime}(t)\right|^{2} d t\right)^{\frac{1}{2}} \\
\leq & {\left[L_{1} \frac{T}{\pi}\left(\frac{T}{2 \pi}\right)^{2(n-1)}+\sum_{k=2}^{n+1} L_{k}\left(\frac{T}{2 \pi}\right)^{2 n-k}+B\left(\frac{T}{\pi}\right)^{2}\left(\frac{T}{2 \pi}\right)^{2(n-1)}\right]\left\|Z^{(n)}\right\|_{2}^{2}, }
\end{aligned}
$$

which together with (2.3) and (2.12) implies that

$$
Z^{(n)}(t) \equiv Z^{(n-1)}(t) \equiv \cdots \equiv Z^{\prime}(t) \equiv Z(t) \equiv 0 \quad \text { for all } t \in R
$$


Therefore, Eq. (1.3) has at most one $T$-periodic solution. The proof of Lemma 2.6 is now completed.

\section{Main results}

Theorem 3.1 Let (S1), (S2) (or (S3)) hold and let either $f_{n+1}(x) \equiv 0$ or $\left|f_{n+1}(x)\right| \geq \alpha^{*}>0$ for all $x \in R$, where $\alpha^{*}$ is constant. Then Eq. (1.3) has a unique T-periodic solution.

Proof From Lemma 2.6, we have obtained that Eq. (1.3) has at most one $T$-periodic solution. Thus, to prove Theorem 3.1, it suffices to show that Eq. (1.3) has at least one $T$-periodic solution. To do this, we will use Lemma 2.1. Firstly, let us show that (i) in Lemma 2.1 is satisfied, which means there exists $\rho>0$ such that any possible $T$-periodic solution $x$ of (2.5) satisfies

$$
\|x\|_{(2 n-1)}<\rho .
$$

By Lemma 2.5, there exists $C^{*}>0$ such that any possible $T$-periodic solution $x$ of (2.5) satisfies

$$
\left\|x^{(k)}\right\|_{\infty} \leq C^{*}, \quad k=0,1,2, \ldots, n-1 .
$$

If $f_{n+1}(x) \equiv 0$, from (2.5), (2.6) and (2.10), it follows that there exists a constant $C^{* *}$ satisfying

$$
\int_{0}^{T}\left|x^{(2 n)}(t)\right| d t \leq C^{* *}
$$

which together with (2.4) implies the existence of a constant $\rho, \rho>d$ such that (3.1) holds.

If $\left|f_{n+1}(x)\right|>\alpha^{*}>0$, multiplying Eq. (2.5) by $x^{(n+1)}$ and integrating it from 0 to $T$, we obtain

$$
\begin{aligned}
\alpha^{*}\left\|x^{(n+1)}(t)\right\|_{2}^{2}= & \alpha^{*} \int_{0}^{T}\left|x^{(n+1)}(t)\right|^{2} d t \\
\leq & \sum_{k=1}^{n} \sup _{t \in[0, T]} \mid f_{k}\left(x^{(k-1)}(t)\left|\int_{0}^{T}\right| x^{(k)}(t)|| x^{(n+1)}(t) \mid d t\right. \\
& +\sup _{t \in[0, T]}|g(t, x(t))| \int_{0}^{T}\left|x^{(n+1)}(t)\right| d t+\sup _{t \in[0, T]}|e(t)| \int_{0}^{T}\left|x^{(n+1)}(t)\right| d t \\
\leq & \sum_{k=1}^{n} \sup _{t \in[0, T]} \mid f_{k}\left(x^{(k-1)}(t) \mid\left(\int_{0}^{T}\left|x^{(k)}(t)\right|^{2} d t\right)^{\frac{1}{2}}\left(\int_{0}^{T}\left|x^{(n+1)}(t)\right|^{2} d t\right)^{\frac{1}{2}}\right. \\
& +\|g\|_{\infty} \sqrt{T}\left(\int_{0}^{T}\left|x^{(n+1)}(t)\right|^{2} d t\right)^{\frac{1}{2}}+\|e\|_{\infty} \sqrt{T}\left(\int_{0}^{T}\left|x^{(n+1)}(t)\right|^{2} d t\right)^{\frac{1}{2}} \\
\leq & {\left[\sum_{k=1}^{n} \sup _{t \in[0, T]} \mid f_{k}\left(x^{(k-1)}(t) \mid\left\|x^{(k)}\right\|_{2}+\|g\|_{\infty} \sqrt{T}+\|e\|_{\infty} \sqrt{T}\right]\left\|x^{(n+1)}(t)\right\|_{2} .\right.}
\end{aligned}
$$

Therefore, there exists a positive constant $C_{n+1}$ satisfying

$$
\int_{0}^{T}\left|x^{(n+1)}(t)\right|^{2} d t \leq C_{n+1}, \quad \int_{0}^{T}\left|x^{(n+1)}(t)\right| d t \leq \sqrt{T C_{n+1}},
$$


which together with (2.5), (2.6), (2.10) implies (3.2) holds. Thus, from (2.4) and (3.2), we can also show that (3.1) holds.

Now, to show that (ii) in Lemma 2.1 is satisfied, it suffices to remark that

$$
F(x)=\int_{0}^{T}[g(t, x)+e(t)] d t, \quad x \in R .
$$

Hence, from (S1) and $\rho>d$, it results that $F(-\rho) F(\rho)<0$. Then, by Lemma 2.1, we obtain that (1.3) has at least one $T$-periodic solution $x$ satisfying $\|x\|_{(2 n-1)}<\rho$. This completes the proof.

\section{An example}

Example 4.1 Let $n=4, T=\pi, f_{5}(x)=\sin x, f_{k}(x)=e^{\frac{1}{k}} \cos x, k=1,2,3,4, g(t, x(t))=$ $-e^{|\sin 4 t|} x^{3}(t), e(t)=\frac{1}{4} \sin 2 t$. Then

$$
x^{(8)}(t)+\sin \left(x^{(4)}(t)\right) x^{(5)}(t)+\sum_{k=1}^{4} e^{\frac{1}{k}} \cos x^{(k-1)}(t) x^{(k)}(t)-e^{|\sin 4 t|} x^{3}(t)=\frac{1}{4} \sin 2 t
$$

has a unique $\pi$-periodic solution.

Proof By (4.1), we have $n=4, L_{5}=1, L_{k}=e^{\frac{1}{k}}, k=1,2,3,4, T=\pi$, then

$$
L_{1} \frac{T}{\pi}\left(\frac{T}{2 \pi}\right)^{2(n-1)}+\sum_{k=2}^{n+1} L_{k}\left(\frac{T}{2 \pi}\right)^{2 n-k}<\frac{5}{8}<1 .
$$

It is obvious that the assumptions (S1), (S2) hold. Hence, by Theorem 3.1, (4.1) has a unique $\pi$-periodic solution.

Example 4.2 Let $n=4, T=\pi, f_{5}(x)=\sin x, f_{k}(x)=e^{\frac{1}{k}} \cos x, k=1,2,3,4, g(t, x(t))=$ $\arctan x(t), e(t)=\frac{1}{4} \sin 2 t$. Then

$$
x^{(8)}(t)+\sin \left(x^{(4)}(t)\right) x^{(5)}(t)+\sum_{k=1}^{4} e^{\frac{1}{k}} \cos x^{(k-1)}(t) x^{(k)}(t)-\arctan x(t)=\frac{1}{4} \sin 2 t
$$

has a unique $\pi$-periodic solution.

Proof By (4.2), we have $n=4, L_{5}=1, L_{k}=e^{\frac{1}{k}}, k=1,2,3,4, B=1, T=\pi$. Then

$$
L_{1} \frac{T}{\pi}\left(\frac{T}{2 \pi}\right)^{2(n-1)}+\sum_{k=2}^{n+1} L_{k}\left(\frac{T}{2 \pi}\right)^{2 n-k}+B\left(\frac{T}{\pi}\right)^{2}\left(\frac{T}{2 \pi}\right)^{2(n-1)}<\frac{6}{8}<1 .
$$

It is obvious that the assumptions (S1), (S3) hold. Hence, by Theorem 3.1, (4.2) has a unique $\pi$-periodic solution.

Remark 4.1 Obviously, the authors in [1-13] only considered the existence of periodic solutions of a high-order nonlinear differential equation. Although the author in [18] considered the existence and uniqueness of periodic solutions of high-order nonlinear differential equation, the coefficients of $x^{(k)}(t)$ are constants. Hence, the results obtained in 
$[1-13,18]$ and the references cited therein are not applicable to Examples 4.1-4.2. This implies that the results of this paper are essentially new.

\section{Competing interests}

The authors declare that they have no competing interests.

\section{Authors' contributions}

RJ gave the proof of Lemma 2.5 and drafted the manuscript. JS proved Theorem 3.1 and gave two examples to illustrate the effectiveness of the obtained results. All authors read and approved the final manuscript.

\section{Author details}

${ }^{1}$ College of Mathematics and Computer Science, Hunan University of Arts and Science, Changde, Hunan 415000, P.R. China. ${ }^{2}$ College of Mathematics, Physics and Information Engineering, Jiaxing University, Jiaxing, Zhejiang 314001, P.R. China.

\section{Acknowledgements}

The authors would like to express the sincere appreciation to the reviewers for their helpful comments in improving the presentation and quality of the paper. This work was supported by the National Natural Science Foundation of China (grant No. 11201184), the Natural Scientific Research Fund of Hunan Provincial of China (Grant No. 11JJ6006), the Natural Scientific Research Fund of Hunan Provincial Education Department of China (Grant Nos. 11C0916 and 11C0915), and the Natural Scientific Research Fund of Zhejiang Provincial of P.R. China (grant No. LY12A01018).

Received: 30 November 2012 Accepted: 24 February 2013 Published: 20 March 2013

\section{References}

1. Amster, P, Mariani, MC: Oscillating solutions of a nonlinear fourth order ordinary differential equation. J. Math. Anal. Appl. 325, 1133-1141 (2007)

2. Carriao, PC, Faria, LFO, Miyagaki, OH: Periodic solutions for extended Fisher-Kolmogorov and Swift-Hohenberg equations by truncature techniques. Nonlinear Anal. 67, 3076-3083 (2007)

3. Yao, Q: Existence, multiplicity and infinite solvability of positive solutions to a nonlinear fourth-order periodic boundary value problem. Nonlinear Anal. 63, 237-246 (2005)

4. Chaparova, J: Existence and numerical approximations of periodic solutions of semilinear fourth-order differential equations. J. Math. Anal. Appl. 273, 121-136 (2002)

5. Mawhin, J, Zanolin, F: A continuation approach to fourth order superlinear periodic boundary value problems. Topol. Methods Nonlinear Anal. 2, 55-74 (1993)

6. Peletier, LA, Troy, WC: Spatial patterns described by the Fisher-Kolmogorov equation: periodic solutions. SIAM J. Math. Anal. 28, 1317-1353 (1997)

7. Tersian, S, Chaparova, J: Periodic and homoclinic solutions of extended Fisher-Kolmogorov equations. J. Math. Anal. Appl. 260, 490-506 (2001)

8. Ward, JR: Asymptotic conditions for periodic solutions of ordinary differential equations. Proc. Am. Math. Soc. 81 415-420 (1981)

9. Li, F, Li, Y, Liang, Z: Existence and multiplicity of solutions to $2 m$ th-order ordinary differential equations. J. Math. Anal. Appl. 331, 958-977 (2007)

10. Bereanu, C: Periodic solutions of some fourth-order nonlinear differential equations. Nonlinear Anal. 71(1-2), 53-57 (2009)

11. Zhao, C, Chen, W, Zhou, J: Periodic solutions for a class of fourth-order nonlinear differential equations. Nonlinear Anal. TMA 72(3-4), 1221-1226 (2010)

12. Fan, Q, Wang, W, Zhou, J: Periodic solutions of some fourth-order nonlinear differential equations. J. Comput. Appl. Math. 233(2), 121-126 (2009)

13. Xu, M, Wang, W, Yi, X: Periodic solutions for a class of nonlinear $2 n$ th-order differential equations. Nonlinear Anal. 11 3399-3405 (2010)

14. Mawhin, J: Topological Degree Methods in Nonlinear Boundary Value Problems. CBMS Series in Mathematics, vol. 40. Am. Math. Soc., Providence (1979)

15. Liu, B, Huang, L: Existence and uniqueness of periodic solutions for a kind of first order neutral functional differential equations. J. Math. Anal. Appl. 322, 121-132 (2006)

16. Hardy, GH, Littlewood, JE, Polya, G: Inequalities. Cambridge University Press, London (1964)

17. Mawhin, J: Periodic solutions of some vector retarded functional differential equations. J. Math. Anal. Appl. 45 , 588-603 (1974)

18. Zhou, Q, Gong, S: The existence and uniqueness of periodic solutions for some nonlinear nth-order differential equations. Abstr. Appl. Anal. 2012, Article ID 909387 (2012). doi:10.1155/2012/909387 